In this group of dogs, the range of clinical signs is not significantly different between small and large breeds. The prevalences of clinical and clinicopathological findings appears to be similar to those historically reported. Dogs with more clinical signs tend to have higher ALT and ALP activities.

\section{Canine Hba1c: validation of an immunoturbidimetric assay and determination of a reference interval}

\section{Anne Florentine Goemans, lan Ramsey}

University of Glasgow, Glasgow, UK

\section{BACKGROUND}

Haemoglobin A1c ( $\mathrm{HbA1c})$, provides a reliable measure of glycaemic control over 2 to 3 months in human diabetics. In dogs, formation of $\mathrm{HbA1c}$ has been demonstrated; but currently no commercial assays exist for determining canine $\mathrm{HbA1c}$. The validation of such an assay could have significant benefits for the management of canine diabetes.

\section{OBJECTIVE}

To validate a commercially available automated immunoturbidimetric assay for canine $\mathrm{HbA1c}$ and determine a reference interval for canine $\mathrm{HbA1c}$ in a normoglycaemic hospital population.

\section{METHODS}

The specificity of the assay was assessed by inducing $\mathrm{HbA1c}$ in vitro using isolated canine haemoglobin preparations. Imprecision was assessed using manufacturer supplied control materials for between run imprecision. The linearity was assessed by mixing known samples in differing proportions and the effect of anticoagulants by taking paired samples. 7 Samples with known lipid concentrations, triglycerides from $0.68-25.58 \mathrm{mmol} / \mathrm{L}$ and cholesterol from 4.9-11.7 $\mathrm{mmol} / \mathrm{L}$ were measured before and after removal of serum and replacement with $0.9 \%$ saline. The effect of haemolysis was also determined. A reference interval and the effect of age and breed predisposition was determined using EDTA whole blood samples from 60 normoglycaemic non-anaemic hospitalised animals. Measured values were expressed in terms of mmol of $\mathrm{HbA1c}$ per mol of haemoglobin.

\section{RESULTS}

$\mathrm{HbA1c}$ increased proportionally with glucose concentration in vitro. The between run imprecision was 5.9\% (3\% and 8.8\%). The assay was linear within the expected working range. EDTA and heparin can be used interchangeably for $\mathrm{HbA1c}$ measurement. Measured $\mathrm{HbA1c}$ concentrations were affected by moderate to severe lipaemia and by haemolysis. The reference interval for $\mathrm{HbA1c}$ was 9-18.5 mmol/ mol, with a mean of $14.3 \mathrm{mmol} / \mathrm{mol} \mathrm{HbA} 1 \mathrm{c}$ and a range of 9 to $19 \mathrm{mmol} / \mathrm{mol}$. There was no apparent effect of age or breed on $\mathrm{HbA} 1 \mathrm{c}$ concentration.

\section{CONCLUSIONS}

The assay evaluated provides a reliable method of canine HbA1c measurement with good analytical performance. Further work to assess the effect of interference from lipaemia and haemolysis is needed.

\section{HbA1c measurements in canine diabetic patients compared to normoglycaemic controls and fructosamine measurements.}

\section{Susanna Spence, Floortje Goemans, Aimee Hope, Ian Ramsey}

University of Glasgow, Glasgow, UK

\section{BACKGROUND}

Diabetes mellitus is a common endocrinopathy in small animal medicine yet complications such as diabetic ketoacidosis and cataracts frequently arise. Fructosamine levels and glucose curves are often used to evaluate insulin therapy in dogs but they are not without their limitations. In contrast, in human medicine, measurement of HbA1c (a form of glycosylated haemoglobin) is used to monitor diabetic stability by providing an indication of glycaemic control over the preceding 2 to 3 months. Moreover $\mathrm{HbA1c}$ concentrations are associated with outcome in human diabetes, whereas fructosamine values are not. Recently, the Siemens DCA Vantage $^{\circledR}$ analyser has been demonstrated to measure $\mathrm{HbA1c}$ reliably and repeatably in canine patients and a reference range has been identified.

\section{OBJECTIVE}

To compare HbA1c measurements in diabetic and non-diabetic patients within a hospital population and to determine the relationship between $\mathrm{HbA} 1 \mathrm{c}$ and fructosamine levels in diabetic patients.

\section{METHODS}

$\mathrm{HbA1c}$ was measured from EDTA samples using the Siemens DCA Vantage ${ }^{\circledR}$ analyser. Three groups were included in this study: an uncontrolled diabetic group (Group 1, $n=26$; which included 3 patients with concurrent hyperadrenocorticism); a normoglycaemic control group (Group 2, n=76); and a normoglycaemic group with hyperadrenocorticism (Group 3, n=5). All groups were age matched. Group 1 was 


\section{Oral presentations}

compared to the two other groups independently using a Mann-Whitney $U$ Test. The relationship between $\mathrm{HbA1c}$ and fructosamine in 13 diabetic patients was evaluated using Pearson correlation.

\section{RESULTS}

Group 1 was statistically different from both Group 2 and Group 3 with a $P$ value $<0.05$. Using Pearson correlation, no significant relationship was found between $\mathrm{HbA1c}$ and fructosamine measurements. Three patients had sequential $\mathrm{HbA1c}$ and fructosamine measured over a ten month period. In two of these patients, $\mathrm{HbA1c}$ and fructosamine measurements decreased with a concurrent marked clinical improvement. In the other patient, who was experiencing a Somogyi overswing, $\mathrm{HbA1c}$ and fructosamine levels initially increased before reducing when the glycaemic control and clinical signs of the patient improved.

\section{CONCLUSIONS}

$\mathrm{HbA1c}$ values are statistically different in uncontrolled diabetic patients compared to a normoglycaemic control population. $\mathrm{HbA} 1 \mathrm{c}$ concentrations are not correlated to fructosamine measurements reflecting the difference in time over which they are created. Sequential HbA1c measurements in 3 patients were in agreement with the clinical course. HbA1c measurements should be further investigated for their correlation with long term glycaemic control and prognosis in canine diabetics.

\section{The diagnosis, treatment and therapeutic monitoring of canine hypothyroidism in primary care practice in the UK (2009-2014)}

\section{Rebecca Jones', Nicholas Bexfield ${ }^{1,2}$, Mark Dunning ${ }^{1,2}$}

1 University of Nottingham, Nottingham, UK

2 Pride Veterinary Centre, Derby, UK

\section{INTRODUCTION}

Canine hypothyroidism is a common endocrinological disease in small animal practice, yet it remains a diagnostic challenge. Many publications provide recommendations on the optimal diagnosis and management, however no studies have examined the methods used by UK veterinary surgeons to diagnose and manage the disease. The aim was to review the approaches used in the diagnosis, treatment and therapeutic monitoring of canine hypothyroidism in UK primary care practice, and establish how closely the methods used follow published recommendations.

\section{METHODS}

Case records of 277 dogs tested for hypothyroidism at four UK primary care veterinary practices were reviewed to determine the signalment, history and presenting signs that influenced the decision to test. Haematological and biochemical abnormalities and the specific endocrinological tests used were also reviewed. It was noted whether potential underlying non-thyroidal illness and drug interference prior to thyroid testing were considered. Where hypothyroidism was diagnosed, the methods used to monitor treatment efficacy were recorded.

\section{RESULTS}

The most common clinical signs prompting thyroid testing were dermatological (77.0\%) and metabolic (61.3\%). Routine haematology and biochemistry profiles were conducted prior to thyroid testing in $64.2 \%$ of cases, and medication which could interfere with thyroid function testing was discontinued in $10.3 \%$ of cases where it was a potential confounding factor. First-line diagnostic testing usually comprised total thyroxine (tT4) $(98.5 \%) \pm$ canine thyrotropin (cTSH) (72.0\%), whereas free thyroxine (fT4) and thyroglobulin autoantibody (TgAA) were generally reserved for secondline testing. One veterinary practice included tT4 measurement on all routine biochemistry profiles. Hypothyroidism was subsequently diagnosed in $23.3 \%$ of cases, all of which were treated with licensed forms of levothyroxinesodium. Bradycardia, anaemia and hypercholesterolaemia were significantly more common $(P<0.05)$ in dogs which tested positive for hypothyroidism than in the whole tested population. The test typically used to evaluate therapeutic adequacy was post-treatment tT4 (80.0\%)

\section{CONCLUSIONS}

These results demonstrate that primary care veterinary surgeons use common signs associated with hypothyroidism to direct initial endocrine testing. Moreover, the published recommendations for the diagnosis and management of canine hypothyroidism are generally being followed. However, routine haematology and biochemistry are often not performed at the time of diagnostic testing, which may overlook confounding factors or comorbidities. Additionally, the impact of drug interference on thyroid function testing was often overlooked. Testing may be improved by placing greater emphasis on bradycardia, anaemia and hypercholesterolaemia as markers of hypothyroidism. 